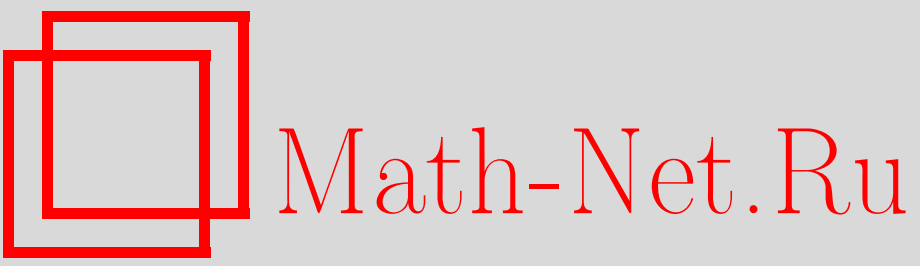

Ю. М. Зиновьев, О критерии Вильсона, ТМФ, 1999, том 118, номер 1, 67-73

DOI: https://doi.org/10.4213/tmf686

Использование Общероссийского математического портала Math-Net.Ru подразумевает, что вы прочитали и согласны с пользовательским соглашением

http://www.mathnet.ru/rus/agreement

Параметры загрузки:

IP : 54.166 .219 .16

26 апреля 2023 г., 15:52:52 


\author{
ТЕОРЕТИЧЕСКАЯ \\ И МАТЕМАТИЧЕСКАЯ \\ ФИЗИКА \\ Том 118, № 1 \\ январь, 1999
}

(C) 1999 г.

Ю. М. Зиновьев ${ }^{*}$

\title{
О КРИТЕРИИ ВИЛЬСОНА
}

Рассматривается $U(1)$-калибровочная теория с действием Виллэна на кубической решетке, аппроксимирующей трех- или четырехмерный тор. Если естественным образом выбрать корреляционные функции, то при стремлении шага решетки к нулю они будут сходиться к корреляционным функциям $\mathbf{R}$-калибровочной электродинамики лишь для специальной зависимости константы связи от числа вершин решетки. Эта специальная зависимость определяется выбором системы корреляционных функций. При всякой другой зависимости непрерывный предел корреляционных функций является вырожденным. Критерий Вильсона для удержания заряженных частиц выполнен для $\mathbf{R}$-калибровочной электродинамики на торе. При стремлении радиуса первоначального тора к бесконечности корреляционные функции сходятся к корреляционным функциям $\mathbf{R}$-калибровочной евклидовой электродинамики.

Проблему удержания кварков Вильсон [1] связал с изучением корреляционных функций для чисто калибровочных теорий на решетке, в которых отсутствуют поля материи. Основная часть работы [1] посвящена $U(1)$-калибровочной теории с периодическими граничными условиями. Критерий Вильсона [1] для удержания кварков выполнен, если для больших замкнутых петель $Г$ корреляционная функция $\langle\exp \{i \theta(\Gamma)\}\rangle$ имеет вид $\exp \{-a \Sigma(\Gamma)\}$, где $\Sigma(\Gamma)$ - минимальная площадь поверхности с границей $\Gamma$.

Настоящая работа посвящена случаю, когда калибровочной группой является аддитивная группа $U(1)=\mathbf{R} /(2 \pi \mathbf{Z})$. Пусть $h(\theta)$ - вешественная, дважды дифференцируемая, четная, периодическая функция с периодом $2 \pi$. Наиболее интересными случаями являются функция энергии Вильсона $h(\theta)=1-\cos \theta[1]$ и функция энергии Виллэна $h_{\beta}[2]$, для которой

$$
\exp \left\{-\beta h_{\beta}(\theta)\right\}=c_{\beta} \sum_{n=-\infty}^{\infty} \exp \left\{-\beta(\theta-2 \pi n)^{2} / 2\right\},
$$

где $\beta>0$ и постоянная $c_{\beta}$ выбрана таким образом, чтобы правая часть равнялась единище при $\theta=0$. Функция Виллэна, как и функция Вильсона, периодична и имеет в нуле нуль второго порядка. Далее мы используем функцию Виллэна.

Пусть $e_{i}, i=1, \ldots, d,-$ стандартные единичные векторы в $\mathbf{R}^{d}$ и $p$ - неотрицательное целое число, меньшее $d . p$-Клетки с началом в $\mathbf{m} \in \mathbf{Z}^{d}$ - это формальные символы $\left(\mathbf{m} ; e_{i_{1}}, \ldots, e_{i_{p}}\right)$, где единичные векторы различны.

\footnotetext{
* Математический институт им. В. А. Стеклова РАН, Москва, Россия
} 
Пусть $G$ - одна из трех абелевых групп: $\mathbf{Z}, \mathbf{R}$ или $U(1)=\mathbf{R} /(2 \pi \mathbf{Z}) \cdot p$-Коцепь с коэффициентами в $G$ - это $G$-значная функция на $p$-клетках $f\left(\mathbf{m} ; e_{i_{1}}, \ldots, e_{i_{p}}\right) \equiv f_{i_{1} \ldots i_{p}}(\mathbf{m})$, которая антисимметрична при перестановках индексов $i_{1}, \ldots, i_{p}$. Для целых чисел $N_{1}$ и $N_{2}$ рассмотрим в $\mathbf{Z}^{d}$ куб $\Lambda=\left\{\mathbf{m} \in \mathbf{Z}^{d}: N_{1} \leqslant m_{i} \leqslant N_{2}, i=1, \ldots, d\right\}$. Периодическими граничными условиями называются соотношения

$$
f_{i_{1} \ldots i_{p}}\left(m_{1}, \ldots, m_{j}+N, \ldots, m_{d}\right)=f_{i_{1} \ldots i_{p}}(\mathbf{m})
$$

где $N=N_{2}-N_{1}+1, j=1, \ldots, d$ и $\mathbf{m}=\left(m_{1}, \ldots, m_{d}\right) \in \mathbf{Z}^{d}$. Для периодических граничных условий мы определим граничный оператор

$$
(\partial f)_{i_{1} \ldots i_{p-1}}(\mathbf{m})=\sum_{\epsilon=0,1} \sum_{i_{0}=1}^{d}(-1)^{\epsilon+1} f_{i_{0} i_{1} \ldots i_{p-1}}\left(\mathbf{m}-\epsilon e_{i_{0}}\right)
$$

и кограничный оператор

$$
\left(\partial^{*} f\right)_{i_{1} \ldots i_{p+1}}(\mathbf{m})=\sum_{\epsilon=0,1} \sum_{k=1}^{p+1}(-1)^{\epsilon+k} f_{i_{1} \ldots \hat{i_{k}} \ldots i_{p+1}}\left(\mathbf{m}+\epsilon e_{i_{k}}\right) .
$$

Для $p$-коцепей с коэффициентами в $\mathbf{Z}$ или $\mathbf{R}$ скалярное произведение определяется следуюшим образом:

$$
(f, g)=\sum_{i_{1}<\cdots<i_{p}} \sum_{\mathbf{m} \in \Lambda} f_{i_{1} \ldots i_{p}}(\mathbf{m}) g_{i_{1} \ldots i_{p}}(\mathbf{m}) .
$$

С помощью функции энергии $h_{\beta}$ и 1 -коцепи $\theta$ на $\Lambda$ с коэффициентами в группе $U(1)=$ $\mathbf{R} /(2 \pi \mathbf{Z})$ построим 2-коцепь $h\left(\partial^{*} \theta\right)$ на $\Lambda$ с вешественными коэффициентами. Эта 2-коцеп для любых индексов $i_{1}<i_{2}$ определяется следующим соотношением:

$$
\left(h_{\beta}\left(\partial^{*} \theta\right)\right)_{i_{1} i_{2}}(\mathbf{m}) \equiv h_{\beta}\left(\left(\partial^{*} \theta\right)_{i_{1} i_{2}}(\mathbf{m})\right) .
$$

Через 1 мы обозначим 2-коцеп, определенную равенством $(\mathbf{1})_{i_{1}} i_{2}(\mathbf{m})=1$ для любых индексов $i_{1}<i_{2}$.

Для параллелепипеда $\Lambda \subset \mathbf{Z}^{d}$ и функции энергии $h_{\beta}$ состояние Гиббса при обратной температуре $\beta$ определяется следуюшим образом:

$$
\langle F\rangle_{\Lambda, \beta}=Z^{-1}\left\{\prod_{\mathbf{m} \in \Lambda ; i=1, \ldots, d} \int_{-\pi}^{\pi} d \theta_{i}(\mathbf{m})\right\} F(\theta) \exp \left\{-\beta\left(h_{\beta}\left(\partial^{*} \theta\right), \mathbf{1}\right)\right\} .
$$

Здесь $\theta$-это 1-коцепь на $\Lambda$ с коэффициентами в $U(1)=\mathbf{R} /(2 \pi \mathbf{Z})$, коцепь $\theta$ удовлетворяет периодическим граничным условиям, мера $d \theta_{i}(\mathbf{m})$ - это мера Лебега на $[-\pi, \pi], Z-$ постоянная нормировки и $F-$ функция переменных $\theta_{i}(\mathbf{m})$.

В этой работе рассматриваются функция энергии Виллэна и периодические граничные условия. Мы будем иметь дело с корреляционными функциями $\langle\exp \{i(j, \theta)\}\rangle_{\Lambda, \beta}$, где $j$ - это 1-коцепь на $\Lambda$ с целыми коэффициентами и $i$ - мнимая 
единица. Скалярное произведение $(j, \theta)$ не определено для 1-коцепи $\theta$ на $\Lambda$ с коэффициентами в $U(1)=\mathbf{R} /(2 \pi \mathbf{Z})$, но выражение $\exp \{i(j, \theta)\}$ определено корректно. Легко показать, что $\langle\exp \{i(j, \theta)\}\rangle_{\Lambda, \beta}=0$, если $j \neq \partial \phi$ для какой-либо 2-коцепи $\phi$ на $\Lambda$ с целыми коэффициентами (см., например, [3]). В силу периодических граничных условий мы можем отождествить противоположные вершины куба $\left[N_{1}, N_{2}+1\right]^{\times d}$ и получить решетку $\mathbf{T}_{N}^{d}$, аппроксимируюшую тор $\mathbf{T}^{d}$ радиуса $R$, где $N=N_{2}-N_{1}+1$. Удовлетворяющие периодическим граничным условиям $p$-коцепи с коэффициентами в абелевой группе $G=\mathbf{Z}, \mathbf{R}$ или $U(1)=\mathbf{R} /(2 \pi \mathbf{Z})$ образуют абелеву группу $C^{p}\left(\mathbf{T}_{N}^{d}, G\right)$.

Пусть $f_{i_{1} \ldots i_{p}}(\mathbf{x})$ - коэффициенты вешественной гладкой дифференциальной $p$-формы на торе $\mathbf{T}^{d}$. Скалярное произведение дифференциальных форм подобно скалярному произведению (4). Мы определим внешнюю производную дифференциальной формы следующим образом:

$$
(d f)_{i_{1} \ldots i_{p+1}}(\mathbf{x})=\sum_{k=1}^{p+1}(-1)^{k+1} \frac{\partial}{\partial x_{i_{k}}} f_{i_{1} \ldots \hat{i_{k}} \ldots i_{p+1}}(\mathbf{x})
$$

Определим сопряженный ей оператор

$$
\left(d^{*} f\right)_{i_{1} \ldots i_{p-1}}(\mathbf{x})=-\sum_{i_{0}=1}^{d} \frac{\partial}{\partial x_{i_{0}}} f_{i_{0} i_{1} \ldots i_{p-1}}(\mathbf{x}) .
$$

Для любой дифференциальной $p$-формы зададим целочисленную $p$-коцепь на $\mathbf{T}_{N}^{d}$

$$
\left(f_{N, b}\right)_{i_{1} \ldots i_{p}}(\mathbf{m})=\left[N^{b} f_{i_{1} \ldots i_{p}}\left(2 \pi R N^{-1} \mathbf{m}\right)\right]
$$

где $N=N_{2}-N_{1}+1, b$ - строго положительное целое число и $[r]$ - целая часть вещественного числа $r$.

Из определений $(2),(7)$ и (8) следует, что

$$
\lim _{N \rightarrow \infty}(2 \pi R)^{-1} N^{-b+1}\left(\partial f_{N, b}\right)_{i_{1} \ldots i_{p-1}}(\mathbf{m}(N))=\left(d^{*} f\right)_{i_{1} \ldots i_{p-1}}(\mathbf{x})
$$

где вектор $\mathbf{m}(N)$ таков, что $2 \pi R N^{-1} \mathbf{m}(N)$ стремится к $\mathbf{x}$ при $N \rightarrow \infty$.

ПРЕДЛОЖЕнИЕ 1. Пусть корреляиионная функиия $\left\langle\exp \left\{i\left(\partial \phi_{N, b}, \theta\right)\right\}\right\rangle_{\mathbf{T}_{N}^{d}, \beta}$ задана равенством (5). Тогда для любой вещественной гладкой дифференциальной 2-формиц ф на торе $\mathbf{T}^{d}$ такой, что $d^{*} \phi \not \equiv 0$, и для любих чисел $\beta_{0}>0, \gamma<d+2 b$

$$
\lim _{N \rightarrow \infty}\left\langle\exp \left\{i\left(\partial \phi_{N, b}, \theta\right)\right\}\right\rangle_{\mathbf{T}_{N}^{d}, \beta_{0} N^{\gamma}}=0 .
$$


ДОКАЗАТЕЛЬСТВО. Пусть циклы $z_{1}, \ldots, z_{g}$ образуют базис группы 2-циклов $Z_{2}\left(\mathbf{T}_{N}^{d}, \mathbf{Z}\right)$, которая является ядром гомоморфизма $\partial: C^{2}\left(\mathbf{T}_{N}^{d}, \mathbf{Z}\right) \rightarrow C^{1}\left(\mathbf{T}_{N}^{d}, \mathbf{Z}\right)$. Симметричная $(g \times g)$-матрица $\Omega_{i j}=\left(z_{i}, z_{j}\right)$ положительно определена и обратима. Введем дуальный базис

$$
\bar{z}_{i}=\sum_{j=1}^{g} \Omega_{i j}^{-1} z_{j}
$$

Согласно [4] справедливо следуюшее равенство:

$$
\left\langle\exp \left\{i\left(\partial \phi_{N, b}, \theta\right)\right\}\right\rangle_{\mathbf{T}_{N}^{d}, \beta}=W_{\mathbf{T}_{N}^{d}, \beta}\left(\partial \phi_{N, b}\right) \frac{\Theta\left(\left(\phi_{N, b}, \overline{\mathbf{z}}\right) \mid 2 \pi i \beta \Omega^{-1}\right)}{\Theta\left(\mathbf{0} \mid 2 \pi i \beta \Omega^{-1}\right)},
$$

где

$$
W_{\mathbf{T}_{N}^{d}, \beta}\left(\partial \phi_{N, b}\right)=\exp \left\{-\frac{1}{2 \beta}\left\{\left(\phi_{N, b}, \phi_{N, b}\right)-\sum_{i=1}^{g}\left(\phi_{N, b}, z_{i}\right)\left(\phi_{N, b}, \bar{z}_{i}\right)\right\}\right\}
$$

является корреляционной функцией $\mathbf{R}$-калибровочной электродинамики на решетке $\mathbf{T}_{N}^{d}$ (см. работу [5]) и $\Theta-\theta$-функция Римана:

$$
\Theta(\mathbf{y} \mid \omega)=\sum_{\mathbf{m} \in \mathbf{Z}^{g}} \exp \left\{i \pi \sum_{j, k=1}^{g} m_{j} \omega_{j k} m_{k}+2 \pi i \sum_{j=1}^{g} m_{j} y_{j}\right\},
$$

зависяшая от вектора $\mathbf{y} \in \mathbf{C}^{g}$. Симметричная $(g \times g)$-матрица $\omega$ в $(13)$ имеет положительно определенную мнимую часть. В нашем случае $\omega=2 \pi i \beta \Omega^{-1}$.

Из соотношения (11) и определения (13) следует неравенство

$$
\left|\left\langle\exp \left\{i\left(\partial \phi_{N, b}, \theta\right)\right\}\right\rangle_{\mathbf{T}_{N}^{d}, \beta}\right| \leqslant W_{\mathbf{T}_{N}^{d}, \beta}\left(\partial \phi_{N, b}\right) .
$$

В работе [4] вычислен предел

$$
\begin{aligned}
\lim _{N \rightarrow \infty} & N^{-d-2 b}\left\{\left(\phi_{N, b}, \phi_{N, b}\right)-\sum_{i=1}^{g}\left(\phi_{N, b}, z_{i}\right)\left(\phi_{N, b}, \bar{z}_{i}\right)\right\}= \\
& =\frac{1}{(2 \pi R)^{2 d}} \sum_{\mu=1}^{d} \sum_{\mathbf{l} \in \mathbf{Z}^{d} ; l_{1}^{2}+\cdots+l_{d}^{2} \neq 0} \frac{R^{2}}{l_{1}^{2}+\cdots+l_{d}^{2}}\left|\left(d^{*} \phi\right) \tilde{\mu}^{(1)}\right|^{2},
\end{aligned}
$$

где

$$
(f)_{\mu}^{\sim}(\mathbf{l})=\int_{0}^{2 \pi R} d \theta_{1} \cdots \int_{0}^{2 \pi R} d \theta_{d} \exp \left\{i R^{-1} \sum_{k=1}^{d} l_{k} \theta_{k}\right\}(f)_{\mu}(\boldsymbol{\theta}) .
$$

Поскольку $d^{*} \phi \not \equiv 0$, предел (15) является строго положительным числом. Теперь равенство (10) следует из неравенства (14) и равенства (15). Предложение 1 доказано. 
ПРЕДЛОЖЕнИЕ 2. Пусть корреляиионная функиия $\left\langle\exp \left\{i\left(\partial \phi_{N, b}, \theta\right)\right\}\right\rangle_{\mathbf{T}_{N}^{d}, \beta}$ задана равенством (5). Тогда для любой вещественной гладкой дифференчиальной 2-формы фна торе $\mathbf{T}^{d}, d=3,4$, и для любих чисел $\beta_{0}>0, \gamma>d+2 b$

$$
\lim _{N \rightarrow \infty}\left\langle\exp \left\{i\left(\partial \phi_{N, b}, \theta\right)\right\}\right\rangle_{\mathbf{T}_{N}^{d}, \beta_{0} N^{\gamma}}=1
$$

ДокАЗАТЕЛЬСТВо. Согласно предложению 2.4 и предложению 3.4 из работы [4] для любой последовательности $\phi_{N} \in C^{2}\left(\mathbf{T}_{N}^{d}, \mathbf{Z}\right)$ и для любых чисел $\beta_{0}>0, \alpha>d, d=3,4$,

$$
\lim _{N \rightarrow \infty} \Theta\left(\left(\phi_{N}, \overline{\mathbf{z}}\right) \mid 2 \pi i \beta_{0} N^{\alpha} \Omega^{-1}\right)=1 .
$$

Теперь равенство (17) следует из равенств (11), (12), (15) и (18). Предложение 2 доказано.

Правая часть равенства $(15)$ совпадает с $(2 \pi R)^{-d}\left(d^{*} \phi, \mathcal{G}\left(d^{*} \phi\right)\right)$, где $\mathcal{G}$ - оператор Грина для оператора Лапласа-Бельтрами на дифференциальных 1-формах на торе $\mathbf{T}^{d}$. В работе [4] доказано следуюшее утверждение.

ПРЕДЛОЖЕНИЕ 3. Пусть корреляиионная функиия $\left\langle\exp \left\{i\left(\partial \phi_{N, b}, \theta\right)\right\}\right\rangle_{\mathbf{T}_{N}^{d}, \beta}$ задана равенством (5). Тогда для любой вещественной гладкой дифференциальной 2-формы ф на торе $\mathbf{T}^{d}, d=3,4$, радиуса $R$ и для любого числа $g>0$

$$
\lim _{N \rightarrow \infty}\left\langle\exp \left\{i\left(\partial \phi_{N, b}, \theta\right)\right\}\right\rangle_{\mathbf{T}_{N}^{d}, g^{-2}(2 \pi R)^{-d} N^{d+2 b}}=\exp \left\{-\frac{g^{2}}{2}\left(d^{*} \phi, \mathcal{G}\left(d^{*} \phi\right)\right)\right\} .
$$

Важно отметить, что для перехода к непрерывному пределу не сушествует "канонического" соотношения между константой связи и числом вершин в решетке. По-разному выбирая число $b=1,2, \ldots$ в предложениях $1-3$, мы получаем различные системы естественных наблюдаемых и различные соотношения между константой связи и числом вершин в решетке, которые дают единственный невырожденный непрерывный предел. Можно высказать гипотезу о том, что такая же ситуация имеет место и в моделях неабелевых калибровочных полей на решетке.

Рассмотрим корреляционные функции (19) и проверим критерий Вильсона. Подставим в равенство (19) следующую квадратично-суммируемую 2-форму на торе $\mathbf{T}^{d}$ :

$$
\phi=\Theta\left(L_{1}-x_{1}\right) \Theta\left(L_{2}-x_{2}\right) h\left(x_{2}, \ldots, x_{d}\right) d x_{1} \wedge d x_{2},
$$

где функция $\Theta(L-x)$ совпадает со ступенчатой функцией Хевисайда на интервале $(0,2 \pi R]$ и является периодической функцией. Функция $h\left(x_{2}, \ldots, x_{d}\right)$ является гладкой на торе $\mathbf{T}^{d-2}$. Из определений $(2),(4)$ и $(8)$ следует, что выражение $\left(\partial \phi_{N, b}, \theta\right)$ является суммой петлевых сумм Вильсона для прямоугольников с вершинами

$$
\begin{aligned}
& \left(0,0, m_{2}, \ldots, m_{d}\right), \quad\left(\left[L_{1} N(2 \pi R)^{-1}\right], 0, m_{2}, \ldots, m_{d}\right), \\
& \left(\left[L_{1} N(2 \pi R)^{-1}\right],\left[L_{2} N(2 \pi R)^{-1}\right], m_{2}, \ldots, m_{d}\right), \quad\left(0,\left[L_{2} N(2 \pi R)^{-1}\right], m_{2}, \ldots, m_{d}\right),
\end{aligned}
$$


которые сглажены с плотностью $h_{N, b}\left(m_{2}, \ldots, m_{d}\right)$ в направлениях, ортогональных плоскостям прямоугольников. Здесь $[r]$ - целая часть вешественного числа $r$.

Правая часть равенства $(15)$ совпадает с $(2 \pi R)^{-d}\left(d^{*} \phi, \mathcal{G}\left(d^{*} \phi\right)\right)$. Из равенств $(7),(15)$ и (16) следует, что подстановка 2-формы (20) в это выражение дает следующий результат:

$$
\begin{aligned}
\left(d^{*} \phi, \mathcal{G}\left(d^{*} \phi\right)\right)= & (2 \pi R)^{-d} \sum_{\mathbf{l} \in \mathbf{Z}^{d} ; l_{1}^{2}+\cdots+l_{d}^{2} \neq 0} R^{3} \frac{l_{1}^{2}+l_{2}^{2}}{l_{1}^{2}+\cdots+l_{d}^{2}} \times \\
& \times\left|h^{\sim}\left(l_{2}, \ldots, l_{d}\right)\right|^{2} \prod_{k=1,2} l_{k}^{-2}\left|\exp \left\{i R^{-1} L_{k} l_{k}\right\}-1\right|^{2}
\end{aligned}
$$

где мы считаем, что функция $l^{-2}\left|\exp \left\{i R^{-1} L l\right\}-1\right|^{2}$ принимает в точке $l=0$ свое естественное значение $L^{2} R^{-2}$. Для того чтобы получить интеграл для одной петли Вильсона, мы должны взять в качестве плотности $h\left(x_{2}, \ldots, x_{d}\right)=\delta\left(x_{2}-a_{2}\right) \ldots \delta\left(x_{d}-a_{d}\right)$. Тогда $\left|h^{\sim}\left(l_{2}, \ldots, l_{d}\right)\right|=1$ и ряд $(21)$ расходится. С физической точки зрения тонкий контур ( $h$ является $\delta$-образной плотностью) не отличается от размазанного. Возьмем в ряду (21) лишь члены, отвечаюшие $l_{2}=\cdots=l_{d}=0$. Используя наше соглашение о функции $l^{-2}\left|\exp \left\{i R^{-1} L l\right\}-1\right|^{2}$, получаем

$$
\begin{aligned}
\left(d^{*} \phi, \mathcal{G}\left(d^{*} \phi\right)\right) \geqslant & (2 \pi R)^{-d}\left|h^{\sim}(\mathbf{0})\right|^{2} \times \\
& \times\left\{\sum_{\mathbf{l} \in \mathbf{Z}^{2}} R^{4} \prod_{k=1,2} l_{k}^{-2}\left|\exp \left\{i R^{-1} L_{k} l_{k}\right\}-1\right|^{2}-\left(L_{1} L_{2}\right)^{2}\right\}
\end{aligned}
$$

где согласно определению (16)

$$
(h) \sim(\mathbf{0})=\int_{0}^{2 \pi R} d \theta_{2} \ldots \int_{0}^{2 \pi R} d \theta_{d} h\left(\theta_{2}, \ldots, \theta_{d}\right) .
$$

Ряд в правой части неравенства (22) является суммой квадратов модулей коэффициентов Фурье функции $\prod_{i=1,2} \Theta\left(L_{i}-x_{i}\right)$. Из равенства Парсеваля следует, что этот ряд равен $(2 \pi R)^{2} L_{1} L_{2}$. Таким образом,

$$
\begin{aligned}
\left(d^{*} \phi, \mathcal{G}\left(d^{*} \phi\right)\right) \geqslant & (2 \pi R)^{-(d-2)}\left|\int_{0}^{2 \pi R} d \theta_{2} \cdots \int_{0}^{2 \pi R} d \theta_{d} h\left(\theta_{2}, \ldots, \theta_{d}\right)\right|^{2} \times \\
& \times L_{1} L_{2}\left(1-\frac{L_{1} L_{2}}{(2 \pi R)^{2}}\right) .
\end{aligned}
$$

Следовательно,

$$
\begin{aligned}
& \exp \left\{-\frac{g^{2}}{2}\left(d^{*} \phi, \mathcal{G}\left(d^{*} \phi\right)\right)\right\} \leqslant \\
& \leqslant \exp \left\{-\frac{g^{2}}{2}(2 \pi R)^{-(d-2)}\left|\int_{0}^{2 \pi R} d \theta_{2} \cdots \int_{0}^{2 \pi R} d \theta_{d} h\left(\theta_{2}, \ldots, \theta_{d}\right)\right|^{2} \times\right. \\
& \left.\quad \times L_{1} L_{2}\left(1-\frac{L_{1} L_{2}}{(2 \pi R)^{2}}\right)\right\}
\end{aligned}
$$


Неравенство (25) доказьвает, что критерий Вильсона [1] для удержания заряженных частиц выполнен в $\mathbf{R}$-калибровочной электродинамике на торе с коррелящионными функциями (19) для $L_{i} \leqslant \pi R, i=1,2$. Это значит, что критерий Вильсона не работает в $U(1)$-калибровочной теории на решетке с периодическими граничными условиями, для которой он и был изобретен.

В работе [4] доказано, что для дифференциальной 2-формы $\phi$, ограничение любого коэффициента которой $\phi_{i j}\left(x_{1}+\pi R, \ldots, x_{d}+\pi R\right)$ на множество $(-\pi R, \pi R)^{\times d}$ не зависит от радиуса $R$ и имеет компактный носитель, выполнено равенство

$$
\begin{aligned}
\lim _{R \rightarrow \infty} & \lim _{N \rightarrow \infty}\left\langle\exp \left\{i\left(\partial \phi_{N, b}, \theta\right)\right\}\right\rangle_{\mathbf{T}_{N}^{d}, g^{-2}(2 \pi R)^{-d} N^{d+2 b}}= \\
\quad & \exp \left\{-\frac{g^{2}}{2(2 \pi)^{d}} \int_{\mathbf{R}^{d}} \frac{1}{p_{1}^{2}+\cdots+p_{d}^{2}} \sum_{\mu=1}^{d}\left|\left(d^{*} \phi\right) \widetilde{\mu}(\mathbf{p})\right|^{2} d^{d} p\right\},
\end{aligned}
$$

где оператор $d^{*}$ определен равенством $(7)$ и функция $f_{\mu}^{\sim}(\mathbf{p})$ является обычным преобразованием Фурье функции $f_{\mu}(\mathbf{x})$ на евклидовом пространстве $\mathbf{R}^{d}$. Правая часть равенства (26) является коррелящионной функцией $\mathbf{R}$-калибровочной евклидовой электродинамики [6]. Корреляционные функции евклидовой электродинамики позволяют нам вычислить функции Швингера. С помощью обычного аналитического продолжения мы находим [6] обобщенные функции Вайтмана. Эти обобшенные функции также определяют новую вайтмановскую теорию, в которой отсутствует первая аксиома: пробные функции образуют определенное подпространство пространства $S(\mathbf{R})^{\times k}$. С помощью некоторого продолжения этих обобшенных функций на все пробные функции из $S(\mathbf{R})^{\times k}$ мы получаем [6] хорошо известный формализм Гупта-Блейлера для свободного электромагнитного поля. Следовательно, наш непрерывный предел для $U(1)$-калибровочной теории разумен с физической точки зрения.

Благодарности. Работа поддержана Российским фондом фундаментальных исследований (грант № 93-011-147).

\section{Список литературы}

[1] K. G. Wilson. Phys. Rev. D. 1974. V. 10. P. 2445-2459.

[2] J. Villain. J. Phys. 1975. V. 36. P. 581-590.

[3] Ю. М. Зиноввев. ТМФ. 1980. Т. 43. С. 309-322.

[4] Yu. M. Zinoviev. Commun. Math. Phys. 1995. V. 168. P. 227-247.

[5] Ю. М. Зиноввев. ТМФ. 1981. Т. 49. С. 156-163.

[6] Ю. М. Зиновьев. ТМФ. 1982. Т. 50. С. 207-220. 